\title{
Análise da Dinâmica do Modelo IS-MP para a Economia Brasileira Contemporânea
}

\section{Analysis of Dynamics of Model IS-MP for the Contemporary Brazilian Economy}

Celso José Costa Junior

Resumo: Este trabalho tem como objetivo discutir uma nova abordagem para o modelo IS-LM proposto por Romer (2000), no qual a curva LM é substituída por uma curva MP (política monetária do Banco Central). O trabalho foi motivado pela necessidade de um modelo mais coerente com a realidade econômica corrente para previsão e análise de políticas econômicas. Também foi feita uma análise dinâmica do modelo para conhecer seu comportamento em relação a mudanças de equilíbrio. Os dados macroeconômicos usados nos modelos econométricos são do Ipeadata, sendo dados trimestrais indo do primeiro trimestre de 1995 ao quarto trimestre de 2007.

Palavras-chave: Dinâmica macroeconômica. Modelos econométricos. Macroeconomias keynesiana. Política monetária.

Abstract: This work has objective to discuss a new approach for the model IS-LM proposal by Romer (2000), which curved LM is substituted by a curved MP (monetary policy of the Central Bank). The work was motivated by the need of a more coherent model with the current economic reality for forecast and analysis of economic policies. It was also made a dynamic analysis of the model to know its behavior in relation to changes in equilibrium. The datas macroeconomics used in the models econometrics is of Ipeadata, it's being quarterly data going from the first quarter of 1995 to the fourth quarter of 2007.

Keywords: Dynamic macroeconomics. Econometrics models. Keynesian macroeconomics. Monetary policy.

JEL Classification: C52; E10; E14; E50.

Mestre em Economia pela Unesp-Araraquara. Funcionário do Banco do Brasil. E-mail: cjcostaj@yahoo.com.br 


\section{Introdução}

Hoje em dia, existe um grande abismo entre teóricos macroeconomistas e policy makers. Um dos motivos desse grande abismo, deve-se à ruptura que ocorreu no final dos anos 1960 e início dos anos 1970, quando a inflação tornou-se a grande vilã da macroeconomia e o modelo IS-LM não conseguiu dar uma boa explicação ao problema. Os Economistas financeiros e os Economistas do governo vêm usando modelos macroeconométricos em suas previsões e análises de políticas econômicas (MANKIW, 1990). Entretanto, para estudos de políticas econômicas e para o ensino o modelo IS-LM continua sendo muito prático, por sua simplicidade de entendimento. No entanto, o meio acadêmico usa pouco esse modelo em suas pesquisas. Muita coisa mudou na macroeconomia desde o texto de John Hicks "Mr Keynes and 'Classics' a Suggested Interpretation”, de 1937, e nas palavras de Blanchard (2000) podemos perceber a importância desse trabalho:

A formalização do modelo IS-LM por Hicks e Hansen pode não ter capturado exatamente o que Keynes tinha em mente, mas descrevendo uma lista de mercados agregados cada uma com sua curva de oferta e demanda, e resolvendo-os num equilibrio geral, transformaram o que agora é chamado de macroeconomia.

Com a introdução das metas de inflação e com os Bancos Centrais seguindo metas de taxa de juros ao invés de metas de oferta monetária uma das suposições do modelo IS-LM - esse modelo perdeu um pouco de sentido, sendo necessário transformá-lo.

Uma característica desta publicação é a simplicidade, pois um modelo nada mais é do que uma representação abstrata da realidade. E segundo Hall e Lieberman (2003):

Um modelo não tem por objetivo ser idêntico à realidade. Pelo contrário, ele representa o mundo real por meio de uma abstração, algo que é extraído do mundo real e que nos ajuda a entender como ele funciona. Em qualquer modelo, muitos detalhes da vida real são deixados de lado. (HALL; LIEBERMAN, 2003, p. 8).

Todo modelo tem de partir de suposições e interpretações teóricas da realidade, embora para ganhar crédito tenha de ser provado empiricamente. Mesmo não deixando de considerar as críticas de Lucas (1976) aos modelos macroeconométricos: 
Dado que a estrutura de um modelo econométrico consiste de regras de decisões ótimas dos agentes econômicos, e que regras de decisões ótimas variam sistematicamente com mudanças na estrutura relevante para a decisão de um maker, seguindo que qualquer mudança na política alteraria sistematicamente a estrutura do modelo, tais modelos somente teriam uma ocasional significância.

Este trabalho está dividido em mais quatro partes além desta introdução: a parte um relata o modelo IS-MP, iniciando com a descrição do modelo IS-LM, seus problemas e suas vantagens; na parte dois é desenvolvido o modelo econométrico, exibindo todos os resultados estatísticos e os procedimentos para aceitar cada modelo; na parte três, é tratada a forma dinâmica do modelo, na qual é demonstrado o comportamento do modelo em relação às mudanças de equilíbrio que ocorreram nos anos de 2002 a 2004; e, na parte quatro, são discutidas as considerações finais.

\section{Modelo IS-MP}

O modelo IS-LM tem sido o melhor para estudo e análise de políticas econômicas, não obstante, como todo modelo, esse não tem unanimidade. Suas simplificações facilitam a compreensão, mas deixam um pouco a desejar no realismo. As críticas mais comuns são: que o modelo não tem microfundamentos; a suposição de preços rígidos; e o não usar expectativas. Sua maior aceitação ocorreu nos anos 1950 e início dos anos 1960, período de baixa inflação nos Estados Unidos. Todavia, quando a inflação tornou-se um problema - do final dos anos 1960 aos anos 1970 - houve a necessidade de mudar o modelo incorporando a demanda agregada.

Nos últimos anos, foi adotado em vários países do mundo, inclusive no Brasil, o modelo de metas de inflação, em que o Banco Central deixa de ter uma política monetária mais ativa para usar suas ferramentas no combate à inflação. Entre as suposições do modelo IS-LM, há aquela que diz que o Banco Central tem metas de oferta de moeda, o que tornaria o modelo menos realista para o modelo econômico atual.

Para uma adequação a metas de inflação, uma proposta é substituir a curva LM por uma curva MP (política monetária) - curva essa que indica a escolha da meta de taxa de juros real pelo Banco Central. Segundo Romer (2000), a ação do Banco Central de escolha da meta da taxa de juros comporta-se em função de variáveis macroeconômicas, tais como produto e inflação. Duas abordagens são possíveis: a primeira diz que a meta de taxa de juros é função apenas da inflação, e seu equilíbrio estaria 
no cruzamento das curvas IS e MP (Gráfico 1). Como o Banco Central espera obter uma inflação mais baixa, em vista disso, quando a inflação está acima (abaixo) do nível desejado, seria aumentada (diminuída) a meta de taxa de juros para contrair (expandir) o produto (Gráficos 2 e 3); a segunda abordagem estabelece que a meta de taxa de juros seria função da inflação e do produto, e seu equilíbrio também seria encontrado pelo cruzamento dessas curvas (Gráfico 4). As políticas de contração e expansão monetária são demonstradas nos Gráficos 5 e 6. Para esta segunda abordagem, Romer (2000) estabelece que a inclinação seria ascendente, por conseguinte, um aumento (diminuição) no produto elevaria (diminuiria) a inflação, forçando o Banco Central a aumentar (diminuir) a meta de taxa de juros para estabilizar a inflação. Entretanto, o resultado econométrico deste trabalho apresentou uma inclinação descendente, algo aparentemente lógico, pois movimentos da meta da taxa de juros têm correlação negativa com o produto.

$$
\begin{gathered}
r=f(\pi) \\
r=f(Y, \pi)
\end{gathered}
$$

A condição de equilíbrio no mercado de bens (curva IS) é determinada por:

$$
Y=C+I+G+X-M
$$

Onde:

$Y=$ produto

$C=$ consumo das famílias

$I$ = investimento das firmas

$G=$ gasto do Governo

$X=$ exportações

$M=$ importações

Neste trabalho, a curva IS tem variáveis endógenas (C, I e M) juntamente com as exógenas ( $\mathrm{G}$ e X).

Considera-se o consumo sendo função da renda disponível, assim:

$$
C=C_{0}+c^{*} Y_{D}
$$

Já o investimento como função da taxa de juros real:

$$
I=I_{0}-a^{*} r
$$


E as variáveis exógenas: gasto do Governo e exportações. Como:

$$
\begin{aligned}
& G=G_{0} \\
& X=X_{0}
\end{aligned}
$$

E, completando o modelo, temos as importações como função da renda e da taxa de câmbio:

$$
M=M_{0}+b^{*} Y-d^{*} E
$$

Onde:

$$
\begin{aligned}
& C_{0}=\text { consumo autônomo } \\
& C=\text { propensão marginal a consumir } \\
& I_{0}=\text { investimento autônomo } \\
& a=\text { elasticidade do investimento a taxa de juros } \\
& G_{0}=\text { gasto do Governo } \\
& X_{0}=\text { exportações autônomas } \\
& M_{0}=\text { importações autônomas } \\
& b=\text { elasticidade das importações a renda } \\
& d=\text { elasticidade das importações ao câmbio }
\end{aligned}
$$

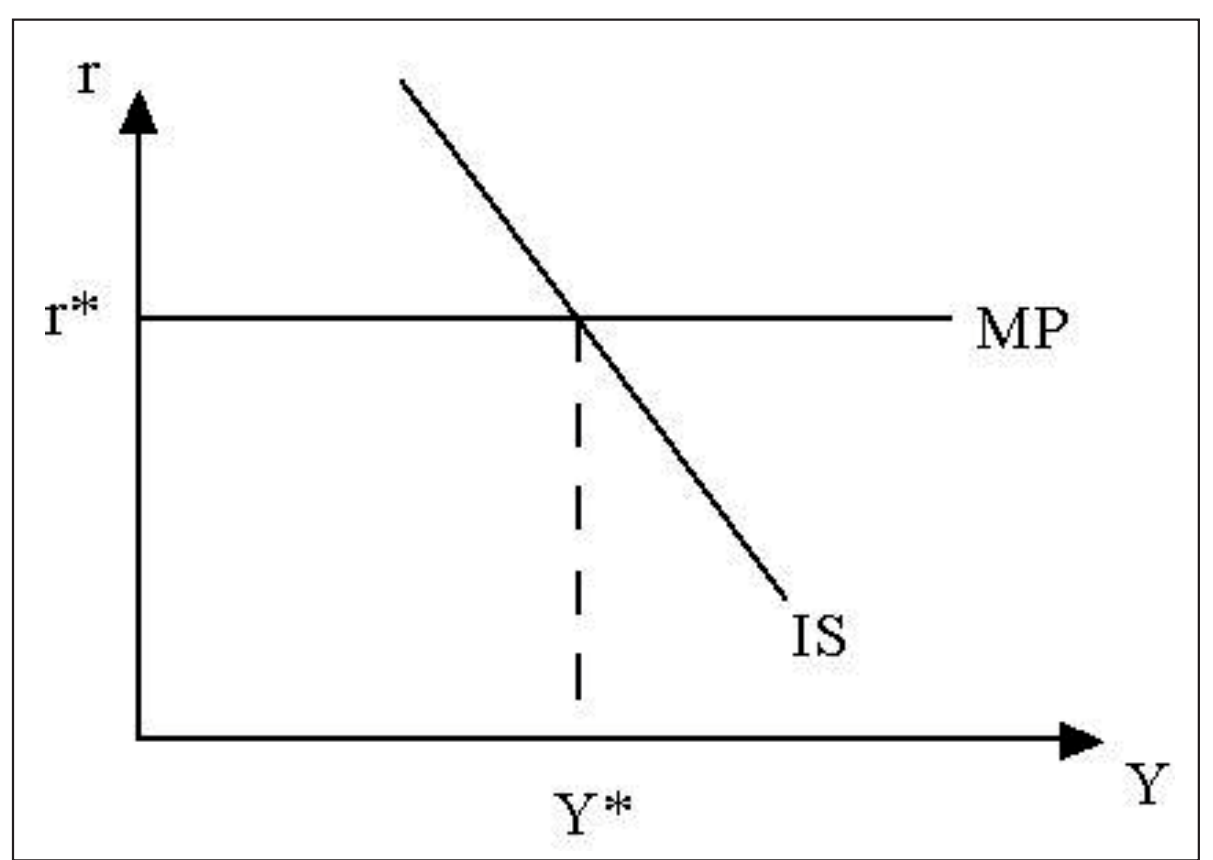

Gráfico 1 - Equilíbrio no modelo IS-MP (primeira abordagem) Fonte: Elaborado pelo autor. 


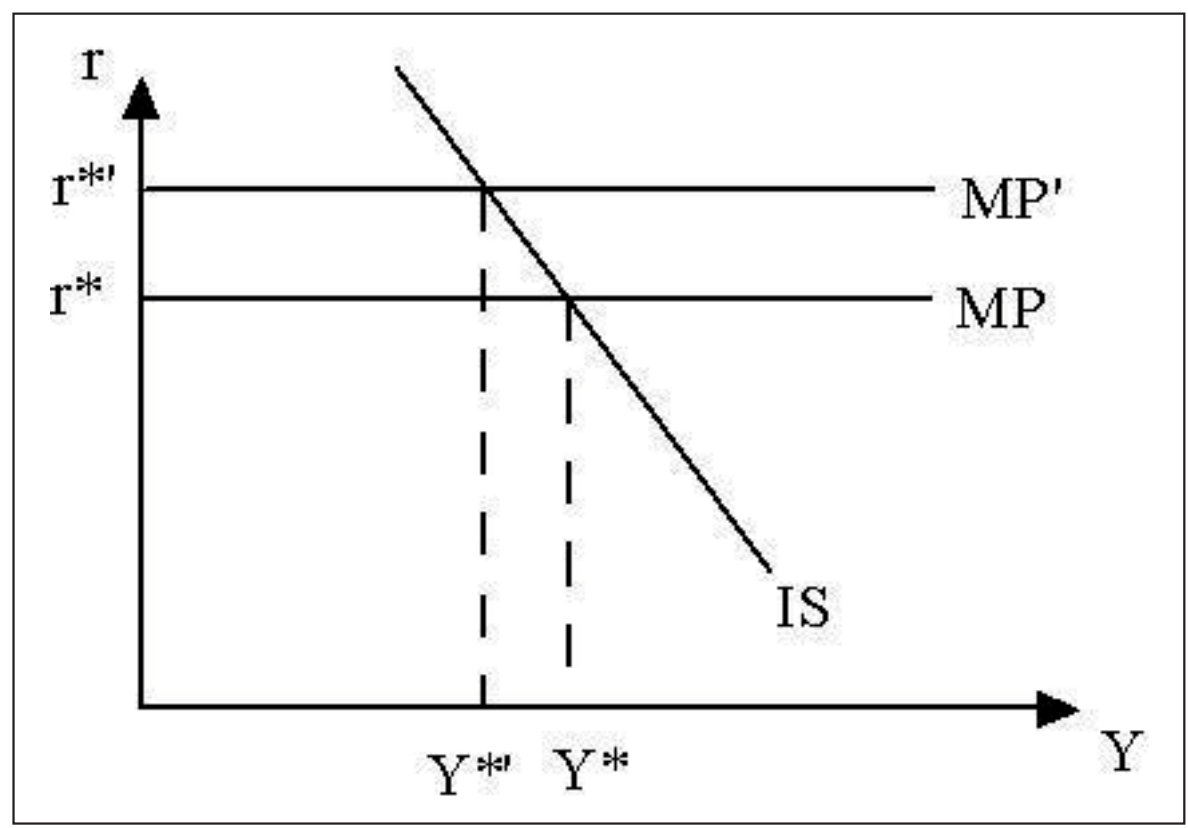

Gráfico 2 - Política de combate à inflação do Banco Central (primeira abordagem) Fonte: Elaborado pelo autor.

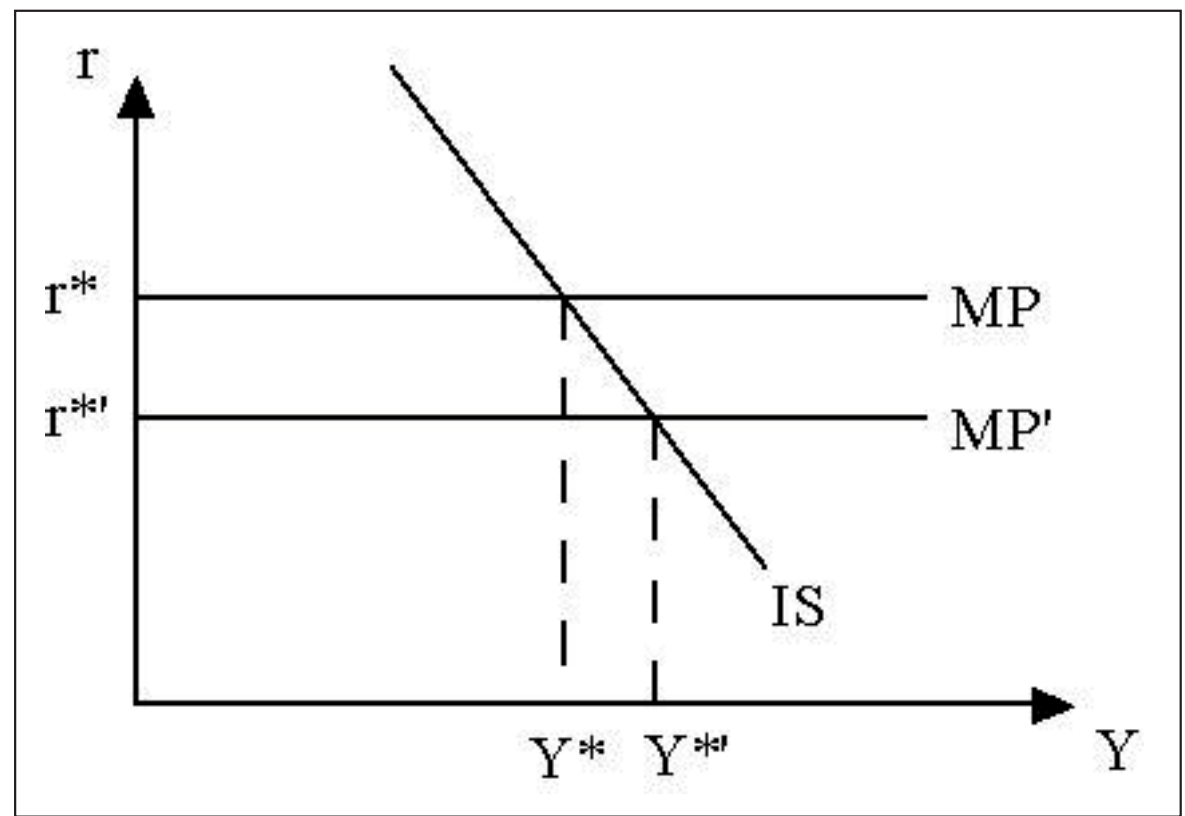

Gráfico 3 - Política expansionista do Banco Central (primeira abordagem) Fonte: Elaborado pelo autor. 


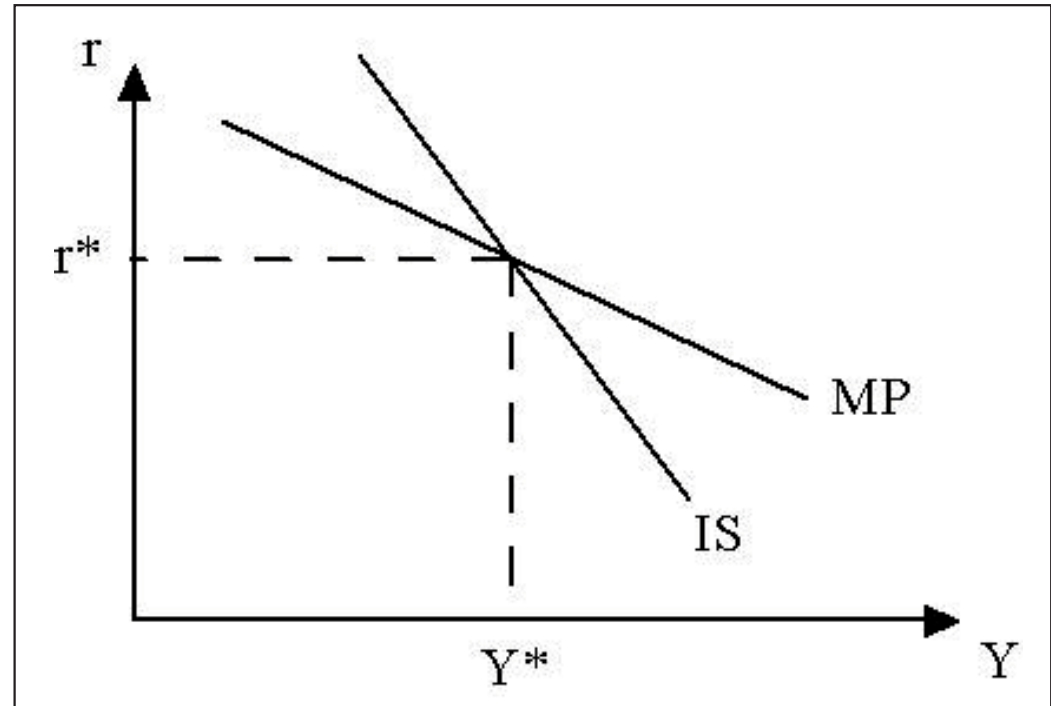

Gráfico 4 - Equilíbrio no modelo IS-MP (segunda abordagem)

Fonte: Elaborado pelo autor.

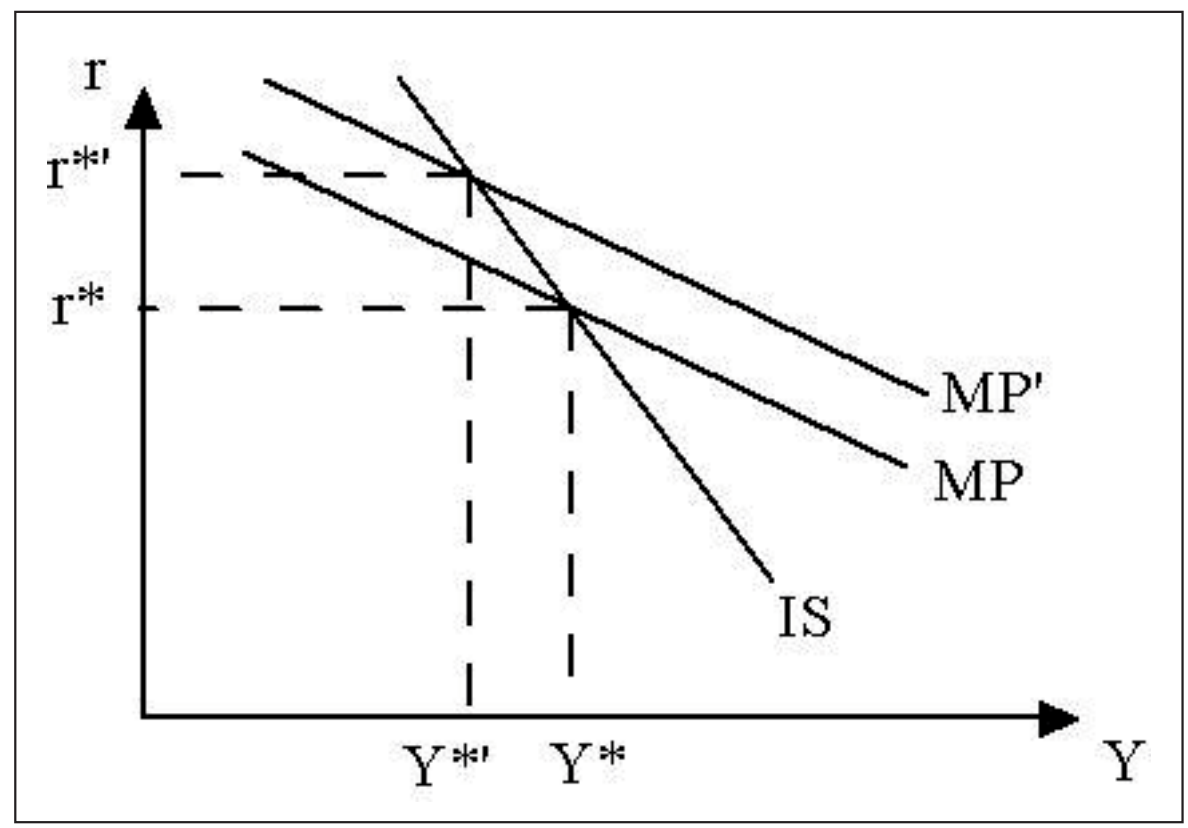

Gráfico 5 - Política de combate à inflação do Banco Central (segunda abordagem) Fonte: Elaborado pelo autor. 


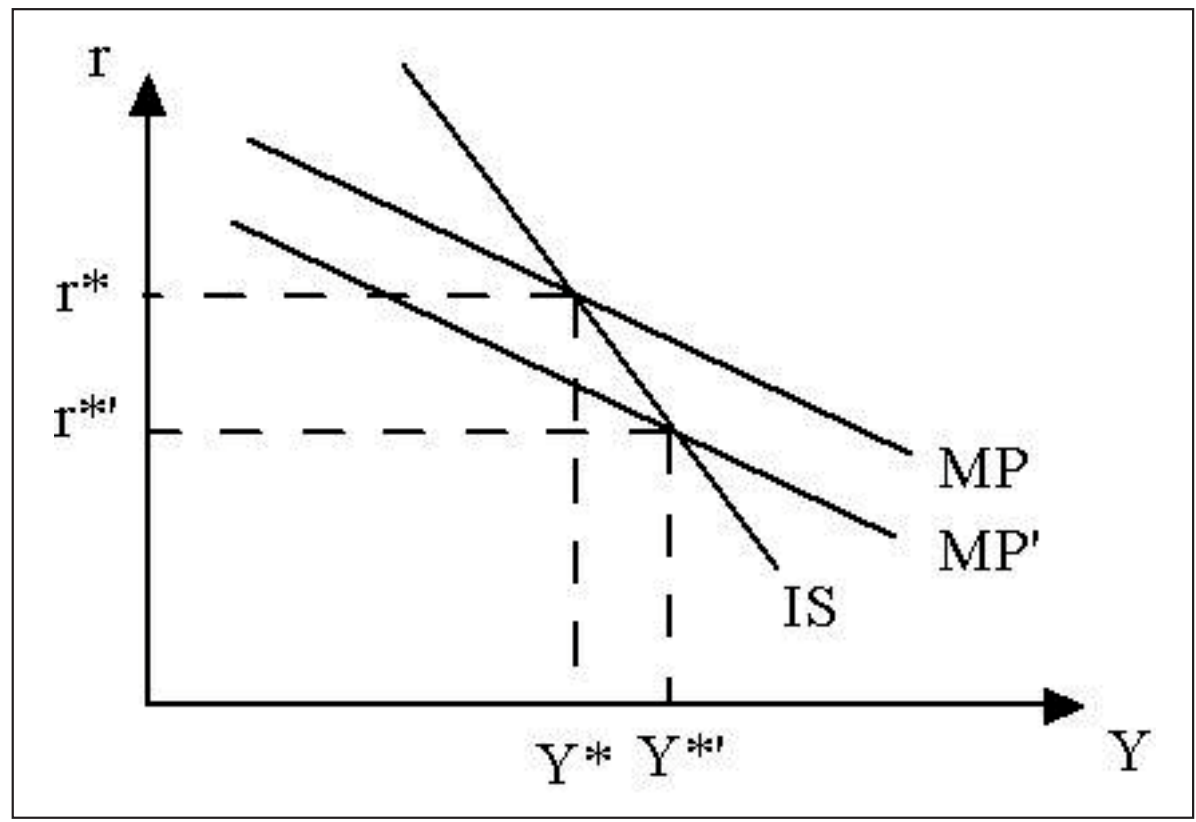

Gráfico 6 - Política expansionista do Banco Central (segunda abordagem) Fonte: Elaborado pelo autor.

\section{Modelo Econométrico}

Partindo do modelo IS-MP discutido na seção anterior, nesta seção é desenvolvido o modelo econométrico através dos métodos dos Mínimos Quadrados Ordinários e dos Mínimos Quadrados de dois estágios (este último aplicado nos modelos quando necessário). Todos os modelos apresentaram resultados satisfatórios, mesmo quando surgiu algum problema com simultaneidade ou possibilidade da regressão ser espúria, os procedimentos adotados na correção desses problemas apresentaram bons resultados.

A primeira regressão trata-se da demanda agregada que seria explicada pelo consumo das famílias; pelo investimento das firmas; pelo gasto do governo; pelas exportações; e pelas importações.

Assim:

$$
D A=\Theta_{0}+\Theta_{1} * C+\Theta_{2} * I+\Theta_{3} * G+\Theta_{4} * X+\Theta_{5} * M+\varepsilon
$$

Onde:

$D A=$ demanda agregada

$C=$ consumo agregado das famílias

$I=$ investimentos das firmas

$G=$ Gasto do Governo 
$X=$ exportações

$M=$ importações

$\varepsilon=$ termo de erro

O resultado da regressão foi:

$$
\begin{gathered}
D A=23875,12+0,85 * C+0,95 * I+0,88 * G+0,91 * X-1,15 * M \\
(4,5) \quad(35,3) \quad(25,3) \quad(27,1) \quad(19,4) \quad(-13,3)
\end{gathered}
$$

Obs: o valor entre parênteses abaixo de cada parâmetro nesta regressão e nas demais é a estatística t.

Resultados dos testes estatísticos:

$\mathrm{R}^{2}$ ajustado $=0,999$

Teste $F(5,46)=42709,08$

Teste Durbin-Watson $=1,25$

Teste Jarque-Bera/Salmon-Kiefer $=0,4$

Teste Breush-Pagan $=3,0$

Analisando o resultado estatístico, temos que a estatística t apresentou um resultado bom para todos os parâmetros, o $\mathrm{R}^{2}$ ajustado forneceu um resultado expressivo indicando um bom ajuste da regressão, e também o teste F bastante alto, e aceitamos as hipóteses de que os erros são normalmente distribuídos e homocedástico, devido aos valores de Jarque-Bera/Salmon-Kiefer e Breush-Pagan, respectivamente. Já o Durbin-Watson apresentou um valor abaixo do tabelado, com isso não podemos aceitar a hipótese nula de não haver autocorrelação, mesmo assim, o modelo não foi alterado, pois mesmo não sendo um MELNV, o estimador continua sendo não viesado e consistente.

O segundo passo foi construir as regressões das variáveis endógenas (C, I e M). Em vista disso, a primeira regressão foi para o consumo, com a renda disponível como variável explicativa:

$$
\begin{gathered}
C=C_{0}+C_{1} * Y_{D}+\varepsilon \\
Y_{D}=Y-T
\end{gathered}
$$

Onde:

$C=$ consumo das famílias

$Y=$ produto

$T=$ arrecadação de tributos pelo Governo

$\varepsilon=$ termo de erro 
Temos o seguinte resultado para essa regressão:

$$
C=28849,15+0,74 * Y_{D}
$$

$\mathrm{R}^{2}$ ajustado $=0,99$

Teste Durbin-Watson $=2,39$

Teste Jarque-Bera/Salmon-Kiefer $=1,71$

Teste Breush-Pagan $=0,88$

Aqui os resultados foram excelentes em todas estatísticas. Portanto, aceitamos que os erros são normalmente distribuídos e homocedástico, e que no modelo não há autocorrelação, logo, os pré-requisitos para a demonstração do Teorema de Gauss-Markov estão satisfeitos e esse modelo é um MELNV.

Em seguida foi realizada a regressão para o investimento, com a taxa básica de juros como variável explicativa:

$$
I=I_{0}+I_{1} * r+\varepsilon
$$

Onde:

$I$ = investimento das firmas

$r=$ taxa básica de juros (Selic)

$\varepsilon=$ termo de erro

A regressão teve como resultado:

$$
I=554095,55-13866,37 * r
$$

$\mathrm{R}^{2}$ ajustado $=0,29$

Teste Durbin-Watson $=0,45$

Teste Jarque-Bera/Salmon-Kiefer $=2,49$

Teste Breush-Pagan $=0,08$

Os valores dos testes estatísticos apresentaram bons resultados, com exceção do teste Durbin-Watson, que forneceu um valor muito baixo, deixando a suspeita de que essa regressão se trate de uma regressão espúria. O procedimento neste caso é tentar provar que as variáveis do modelo são cointegradas, para isso foi feito o teste Johansen (teste não apresentado no trabalho), obtendo bons resultados, dessa forma 
aceitamos que as variáveis são cointegradas. Por conseguinte, aceitamos que os erros são normalmente distribuídos e homocedástico, então, esse modelo é um MELNV.

A próxima regressão é a das importações, com produto e câmbio como variáveis explicativas:

$$
M=M_{0}+M_{1} * Y+M_{2} * E+\varepsilon
$$

Onde:

$M=$ importações

$Y=$ produto

$E=$ câmbio

$\varepsilon=$ termo de erro

Temos como resultado:

$$
\begin{gathered}
M=-50828+0,14 * Y+20003,65 * E \\
(-8,6) \quad(29,1) \quad(6,2)
\end{gathered}
$$

$\mathrm{R}^{2}$ ajustado $=0,97$

Teste $\mathrm{F}(2,49)=864,33$

Teste Durbin-Watson $=0,95$

Teste Jarque-Bera/Salmon-Kiefer $=1,5$

Teste Breush-Pagan $=5,5$

Com os resultados satisfatórios obtidos, aceitamos que os erros são normalmente distribuídos e homocedástico, o único problema aqui é o valor do Durbin-Watson, entretanto, assim como no modelo da demanda agregada, tomamos a decisão de manter o modelo desta forma. O parâmetro de sensibilidade das importações em relação ao câmbio apresentou um valor positivo, algo estranho, pois as importações deveriam cair com a depreciação do câmbio. Todavia, surge uma explicação: o fato de o Brasil ter ficado mais aberto nos últimos anos, exportando mais e também importando mais, a qualquer nível de câmbio.

Terminado o modelo econométrico do lado real, partimos para a regressão do lado monetário, curva MP. Primeiro, trabalhamos com a primeira abordagem, com a taxa de juros em função apenas da inflação. 


$$
r_{M P}=r_{0}+r_{1} * \pi+\varepsilon
$$

Onde:

$r=$ taxa básica de juros (Selic)

$\pi=$ inflação

$\varepsilon=$ termo de erro

O resultado da regressão foi:

$$
r_{M P}=16,34+0,19 * \pi
$$

$$
(15,6) \quad(1,6)
$$

$\mathrm{R}^{2}$ ajustado $=0,04$

Teste Durbin-Watson $=0,54$

Teste Jarque-Bera/Salmon-Kiefer $=5,44$

Teste Breush-Pagan $=0,02$

Os valores dos testes estatísticos foram satisfatórios, com exceção do $\mathrm{R}^{2}$ ajustado e do teste Durbin-Watson, e também o problema do resultado do teste $t$ baixo, indicando que no modelo há multicolinearidade. Mesmo assim, usaremos a equação nos testes dinâmicos deste trabalho.

Restou o modelo econométrico para a segunda abordagem, com a taxa de juros sendo explicada pelo produto e pela inflação:

$$
r_{M P}=r_{0}+r_{1} * Y+r_{2} * \pi+\varepsilon
$$

Onde:

$r=$ taxa básica de juros (Selic)

$Y=$ produto

$\pi=$ inflação

$\varepsilon=$ termo de erro

Chegamos ao resultado:

$$
r_{M P}=\begin{gathered}
22,47-0,0000035 \\
(11,2)
\end{gathered} \quad(-3,3) \quad 0,1 * \pi
$$

$\mathrm{R}^{2}$ ajustado $=0,31$

Teste $\mathrm{F}(2,30)=7,92$

Teste Durbin-Watson $=0,55$ 
Teste Jarque-Bera/Salmon-Kiefer $=1,74$

Teste Breush-Pagan $=1,92$

Os valores dos testes estatísticos foram melhores do que a primeira abordagem, principalmente o $\mathrm{R}^{2}$ ajustado, ainda que o Teste DurbinWatson tenha sido ruim, o resultado do teste de Johansen foi bastante bom (teste omitido neste trabalho). Assim, aceitamos que as variáveis são cointegradas. Portanto, aceitamos que os erros são normalmente distribuídos e homocedástico, e todos pré-requisitos para a demonstração do Teorema de Gauss-Markov estão satisfeitos, em vista disso, esse modelo é um MELNV.

\section{Dinâmica do Modelo}

No modelo IS-MP estático, o equilíbrio geral é obtido no ponto onde tanto o mercado de bens quanto o mercado monetário estão em equilíbrio simultaneamente. O estudo dinâmico fornece uma explicação sobre como um novo equilíbrio é obtido após uma alteração em alguma variável exógena.

Na versão dinâmica do modelo IS-MP, assume-se que no mercado de bens o produto é ajustado de acordo com o excesso de demanda agregada e no mercado monetário pela diferença entre a taxa de juros ótima e a taxa de juros adotada pelo Banco Central.

Então, temos o seguinte sistema de equações diferenciais:

$$
\begin{aligned}
Y^{\prime} & =\alpha(D A-Y) \\
r^{\prime} & =\beta\left(r_{M P}-r\right)
\end{aligned}
$$

A demanda agregada (DA) é obtida pela equação (2.1), juntamente com as equações (2.2), (2.3) e (2.4) e a taxa de juros determinada pelo Banco Central pela equação (2.5) para a primeira abordagem e (2.6) para a segunda.

Para a primeira abordagem, temos o seguinte sistema de equações diferenciais:

$$
\begin{gathered}
Y^{\prime}=\alpha(633239,87+0,88 * G+0,91 * X-0,63 * T-23004,2 * E-0,532 * Y-13173,95 * r) \\
r^{\prime}=\beta(16,34+0,19 * \pi-r)
\end{gathered}
$$


O ponto de equilíbrio ocorre quando $\mathrm{Y}^{\prime}=0$ e $\mathrm{r}^{\prime}=0$. Com $\alpha>0$ e $\beta>0$, temos:

$$
\begin{aligned}
& 633239,87+0,88 * G+0,91 * X-0,63 * T-23004,2 * E-0,532 * Y-13173,95 * r=0 \\
& 16,34+0,19 * \pi-r=0
\end{aligned}
$$

Assim, os pontos de equilíbrio são:

$$
\begin{aligned}
& Y^{*}=785699,68+1,65 * G+1,71 * X-1,18 * T-43240,98 * E-4704,66 * \pi \\
& r^{*}=16,34+0,19 * \pi
\end{aligned}
$$

Para analisar a estabilidade do sistema, foi construída uma matriz Jacobiana.

$$
\begin{gathered}
J=\left[\begin{array}{cc}
-0,532 * \alpha & -13173,95 * \alpha \\
0 & -\beta
\end{array}\right] \\
\operatorname{DET}(J)=0,532 * \alpha * \beta>0 \\
\operatorname{Tr}(J)=-0,532 * \alpha-\beta<0
\end{gathered}
$$

Com o determinante da matriz $\mathrm{J}$ maior que zero e o Traço menor que zero, temos, assim, um sistema estável.

Já para a segunda abordagem, temos outro sistema de equações diferenciais:

$$
\begin{gathered}
Y^{\prime}=\alpha(633239,87+0,88 * G+0,91 * X-0,63 * T-23004,2 * E-0,532 * Y-13173,95 * r) \\
r^{\prime}=\beta(22,47-0,0000035 * Y+0,1 * \pi-r)
\end{gathered}
$$

Aqui também o ponto de equilíbrio ocorre quando $Y^{\prime}=0$ e $r^{\prime}=0$. Com $\alpha>0$ e $\beta>0$, temos:

$157853,56+0,88 * G+0,91 * X-0,63 * T-5751,05 * E-0,532 * Y-3241,35 * r=0$ $22,47-0,0000035 * Y+0,1 * \pi-r=0$ 
Dessa forma, temos os pontos de equilíbrio:

$$
\begin{aligned}
& Y^{*}=693912,43+1,81 * G+1,87 * X-1,3 * T-47333,74 * E-2710,51 * \pi \\
& r^{*}=20,04-0,000006^{*} G-0,000007 * X+0,000005 * T+0,17 * E+0,109 * \pi
\end{aligned}
$$

Também construímos a matriz Jacobiana para a segunda abordagem:

$$
\begin{gathered}
J=\left[\begin{array}{cc}
-0,532 * \alpha & -13173,95 * \alpha \\
-0,0000035^{*} \beta & -\beta
\end{array}\right] \\
\operatorname{DET}(J)=0,486 * \alpha * \beta>0 \\
\operatorname{Tr}(J)=-0,532 * \alpha-\beta<0
\end{gathered}
$$

Aqui também temos um sistema estável.

Os Gráficos 7 e 8 são os diagramas de fase das duas abordagens, onde fica bem visível a estabilidade dos dois sistemas.

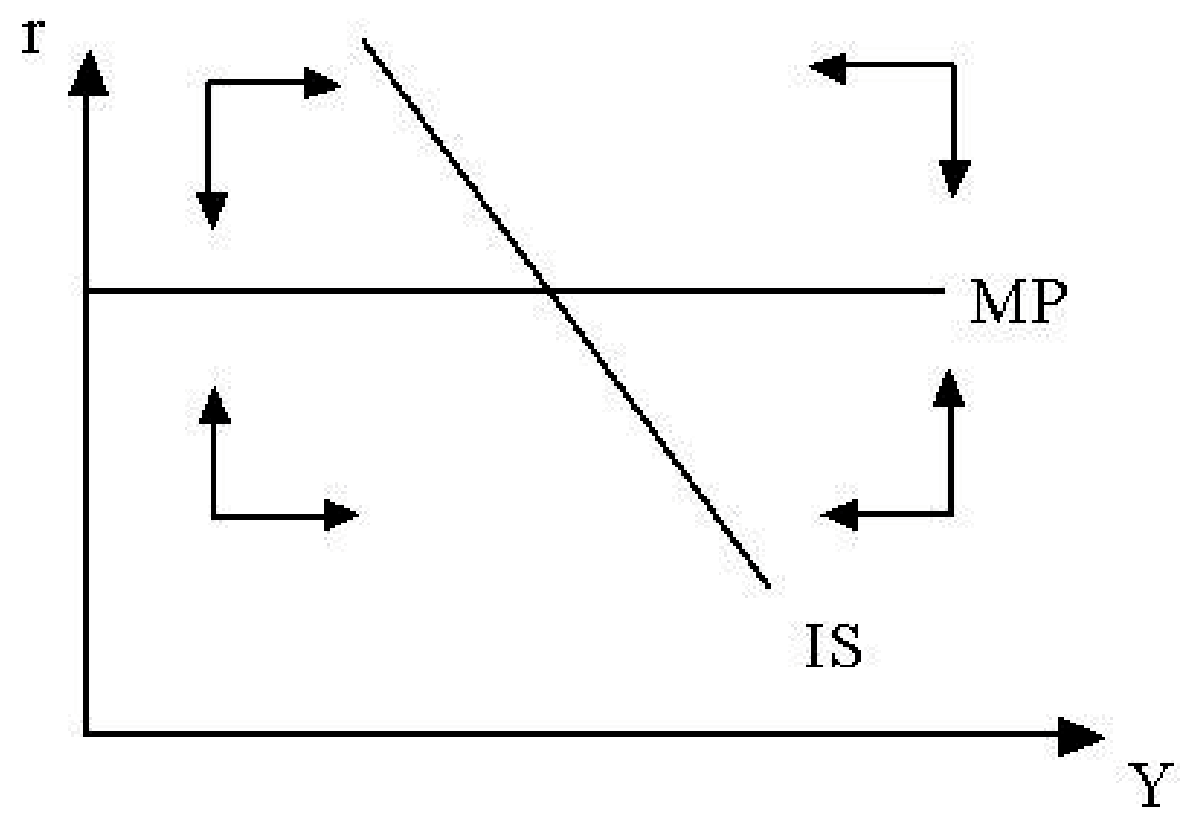

Gráfico 7 - Diagrama de fase do modelo IS-MP (primeira abordagem) Fonte: Elaborado pelo autor. 


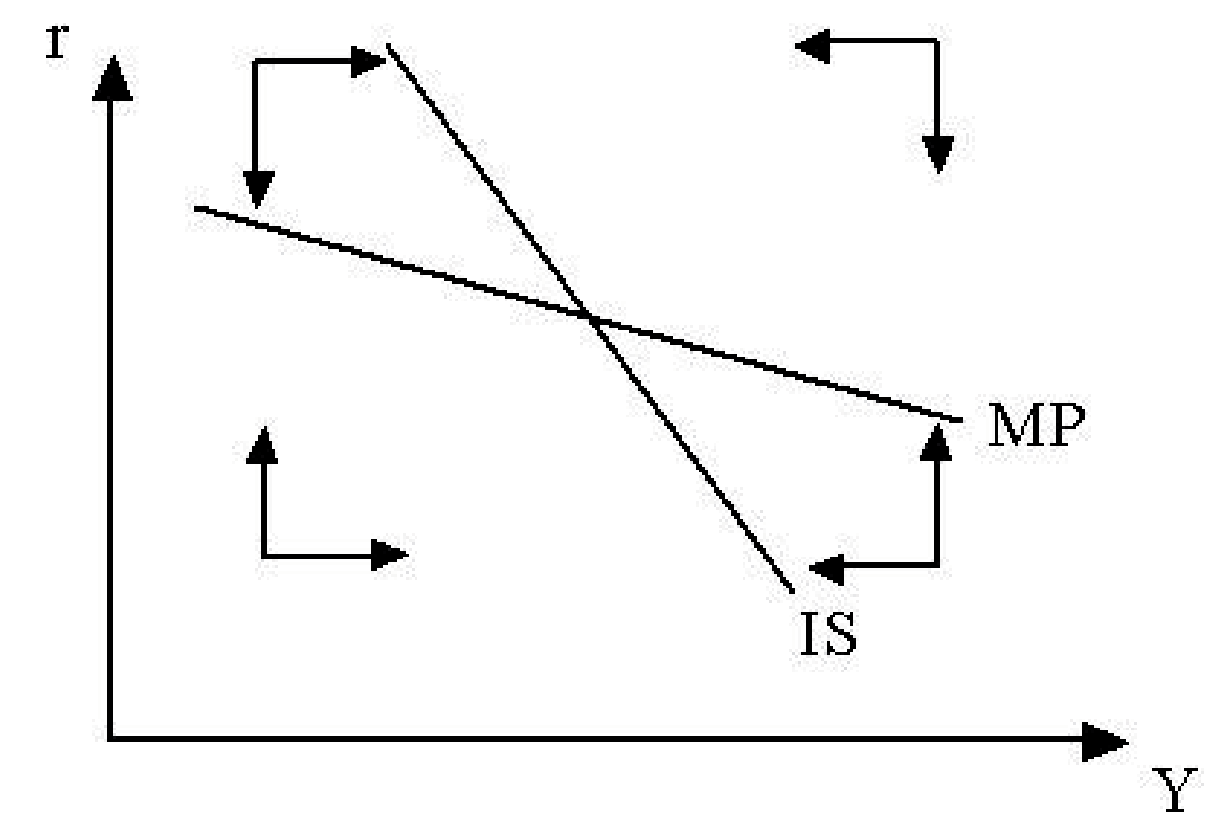

Gráfico 8 - Diagrama de fase do modelo IS-MP (segunda abordagem) Fonte: Elaborado pelo autor.

Para analisar a dinâmica do modelo, foram usados os valores de velocidade de ajustamento de 0,05 e de 0,8 para $\alpha$ e $\beta$, respectivamente, esses valores foram usados por Shone (2002) para descrever um ajustamento instantâneo na taxa básica de juros e gradual para o produto. Esse ajustamento instantâneo para a taxa de juros estaria relacionado com as reuniões do Copom, em que é escolhida uma nova meta de taxa básica de juros (Selic). Também foram escolhidos três momentos para o estudo dinâmico: 1) segundo trimestre de 2002; 2) primeiro trimestre de 2003; e 3) primeiro trimestre de 2004. A ideia seria partir de um momento de inflação controlada, passando para um momento de descontrole, com o Banco Central aumentando a Selic para combater a inflação, e depois um outro momento de volta a um valor estável para inflação. Esses momentos são justificados, pois no final de 2002 o Brasil passava por uma crise política devido à desconfiança do futuro governo Lula. O risco país subiu para 2000 pontos, o câmbio estava na faixa de $4 \mathrm{R} \$$ / US\$ e a expectativa de inflação em torno de $20 \%$ a.a. Para conter essa crise, o Banco Central elevou a Selic no início de 2003 - já no governo Lula - controlando a inflação. Já com a inflação em valores satisfatórios, no início de 2004, o Banco Central começou a diminuir a taxa básica de juros Giambiagi (2005).

No estudo dinâmico, a economia inicia-se em equilíbrio no ponto E', com o aumento da inflação o Banco Central eleva a taxa básica de juros para combatê-la. Para primeira abordagem (Gráfico 9), temos 
que com o aumento da taxa de juros há um movimento de E' para E", instantâneo para a taxa de juros e gradual para o produto. Num segundo momento, com a inflação controlada, o Banco Central diminui a meta da taxa de juros estimulando um aumento na produção - isso é visto no movimento de E" para E"' - chegando ao seu novo valor de equilíbrio. Analisando a segunda abordagem (Gráfico 10), uma elevação da taxa de juros diminui o ritmo de crescimento da renda, entretanto, no equilíbrio E", o produto é superior ao produto em E'. Quando a taxa de juros cai, há a expansão do produto de E" para E"'.

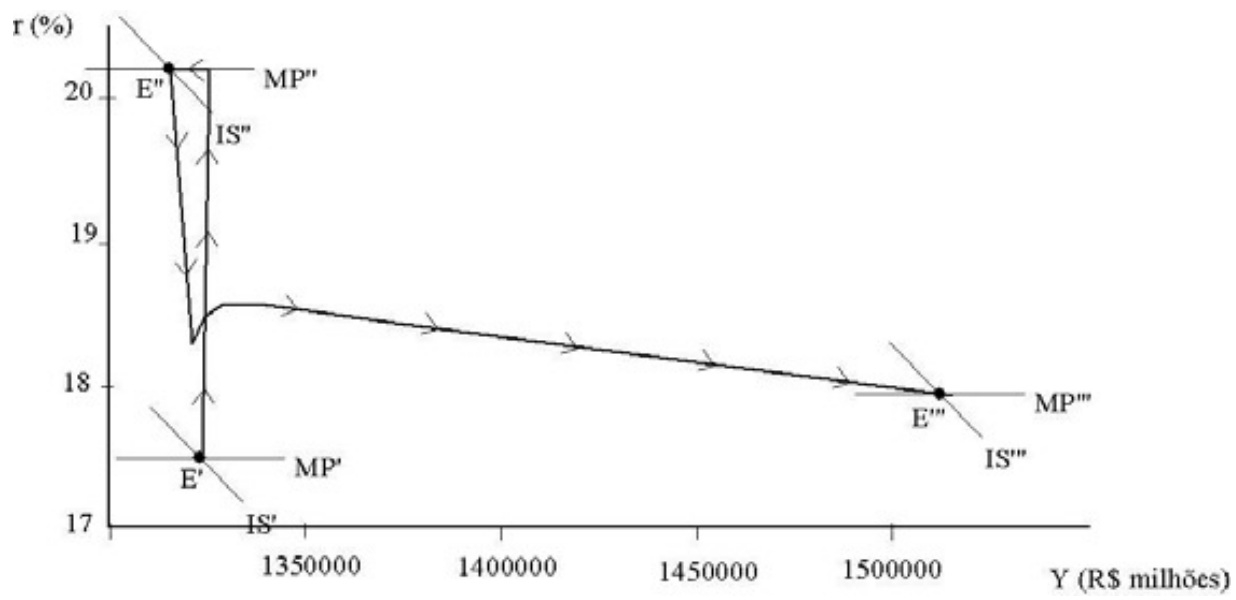

Gráfico 9 - Dinâmica do modelo (primeira abordagem) Fonte: Elaborado pelo autor.

A grande diferença nessas duas abordagens estaria na importância dada ao crescimento do produto pela autoridade monetária. Na primeira abordagem, um aumento expressivo da taxa de juros desacelera a economia, podendo levar até a uma recessão. Nessa abordagem, o pensamento do Banco Central é extremamente conservador, como propõe Rogoff (1985), que diz que um Banco Central tem de ser comandado por um presidente conservador com alta aversão à inflação. A segunda abordagem seria mais indicada para um país em desenvolvimento, como o Brasil, já que esses países, mesmo tendo uma aversão à inflação, dependem do crescimento do produto para aumentar a riqueza per capita. 


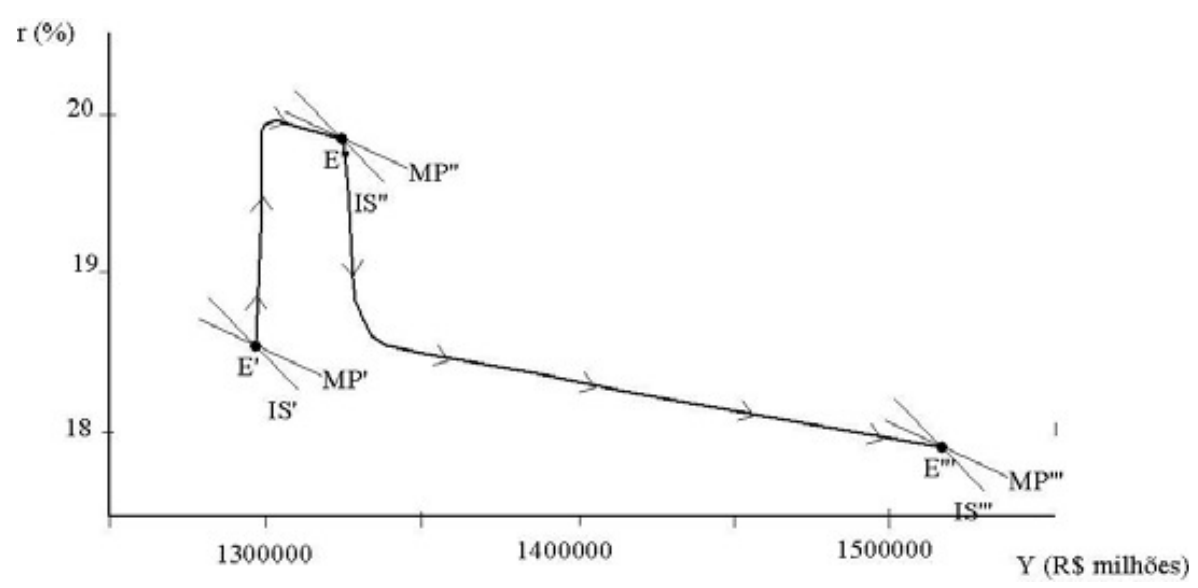

Gráfico 10 - Dinâmica do modelo (segunda abordagem)

Fonte: Elaborado pelo autor.

\section{Considerações Finais}

Nos últimos anos, o modelo IS-LM perdeu aplicabilidade entre os macroeconomistas aplicados, pois esses preferiram usar modelos macroeconométricos. O modelo descrito neste trabalho seria uma solução para esse impasse, pois, ao incorporar as metas de taxa básica de juros, torna o modelo em sintonia com realidade atual. O modelo IS-MP é de fácil aplicabilidade e entendimento. Tornando-se uma ótima ferramenta de previsão e análise de políticas econômicas. A primeira abordagem deveria ser usada por Bancos Centrais de países desenvolvidos, já que sua maior preocupação é o controle inflacionário. Já a segunda abordagem, demonstrou ser mais coerente com países em desenvolvimento, inclusive o Brasil, pois além de ter a inflação como preocupação, o produto demonstrou ser uma variável relevante para a condução da política monetária na curva MP.

\section{Referências}

BLANCHARD, O. What do we know about macroecnomics that Fisher and Wicksell did not? NBER working paper series. Massachusetts: Working paper 7550, feb. 2000.

GIAMBIAGI, F. Rompendo com a ruptura: o governo Lula (2003-2004). In: GIAMBIAGI, F. et al. Economia brasileira contemporânea (1945-2004). Rio de Janeiro: Campus, 2005.

HALL, R. E.; LIEBERMAN, M. Macroeconomia: princípios e aplicações. São Paulo: Thomson, 2003.

LUCAS, R. E. J. Econometric policy evaluation: a critique. In: BRUNNER, K.; MELTZER, A. (Ed.). The phillips curve and labor markets. Amsterdam: North-Holland, 1976. [Carnegie-Rochester Series on Public Policy]. 
MANKIW, N. G. A quick refresher course in macroeconomics. Journal of Economics Literature, v. 28. n. 4, p. 1645-1660, dec. 1990.

ROGOFF, K. The optimal degree of commitment to an intermediate monetary target. Quartely Journal of Economics, v. 100. n. 4, p. 1169-1189, nov. 1985.

ROMER, D. Keynesian macroeconomics without the LM curve. NBER working paper series, Massachusetts: Working paper 7461. jan. 2000.

SHONE, R. Economics dynamics: phase diagrams and their economic application. New York: Cambridge, 2002. 


\section{Apêndice}

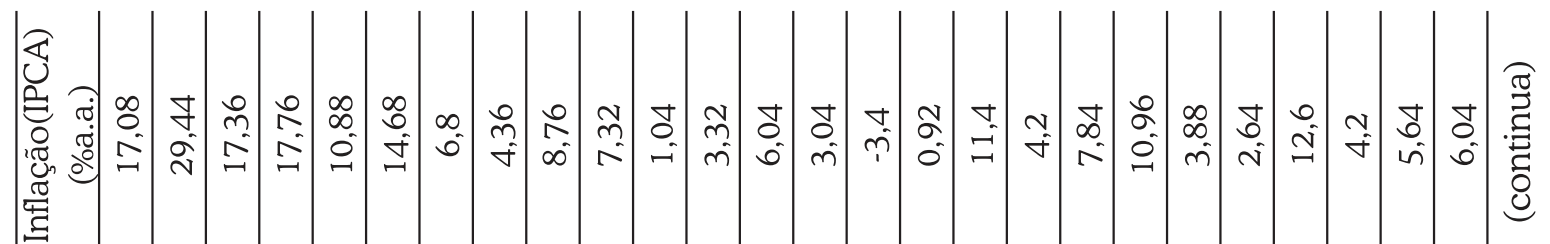

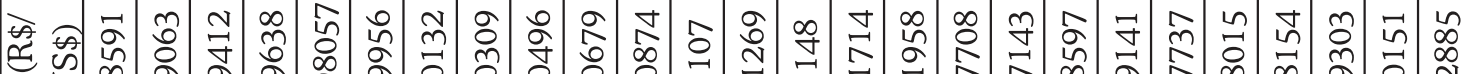

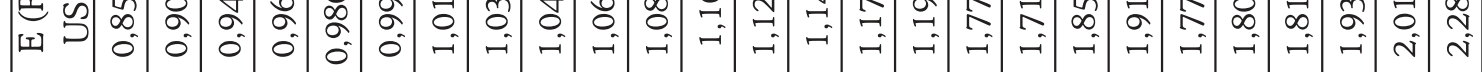

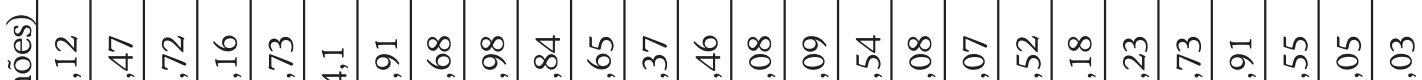

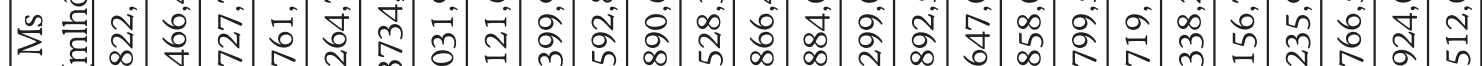

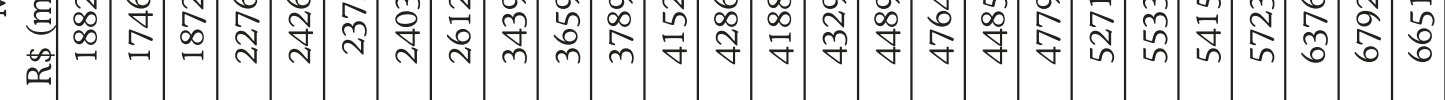

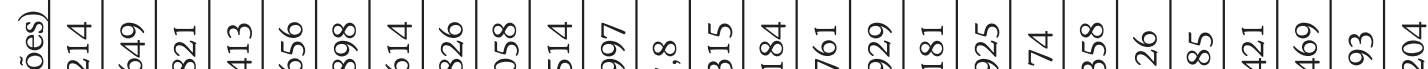

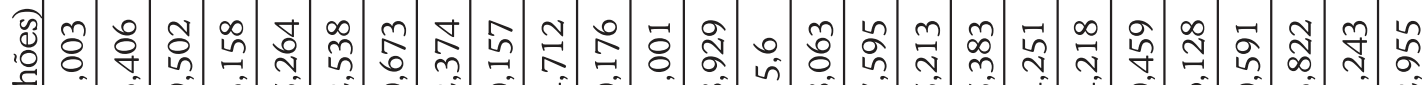

己

ชิ

ఫ ט है 老

ֻี

1

$\rightarrow-\infty$

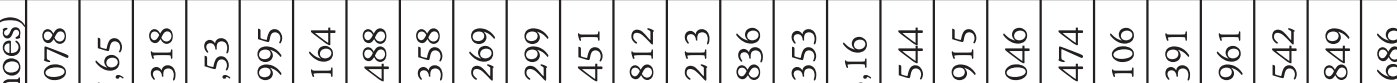

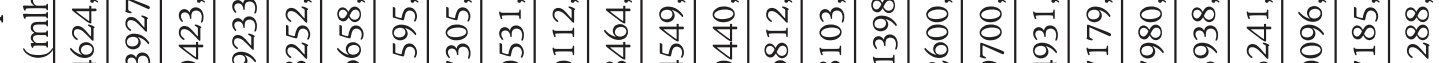

ن

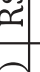

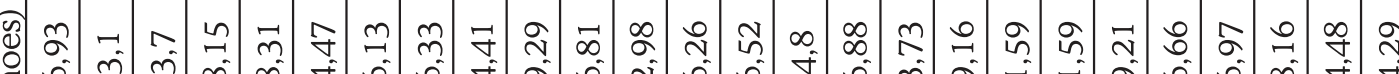

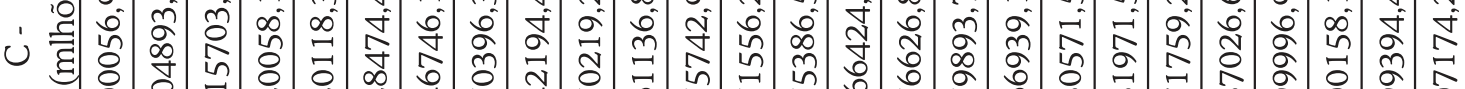

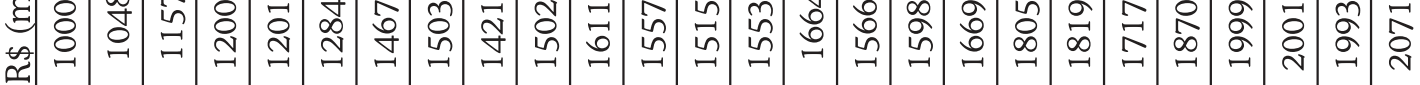

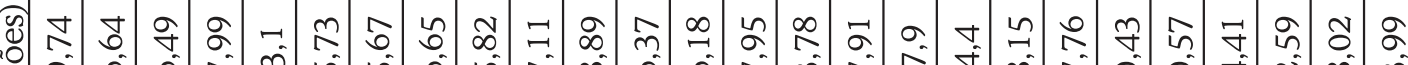

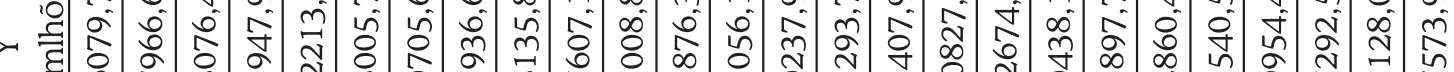

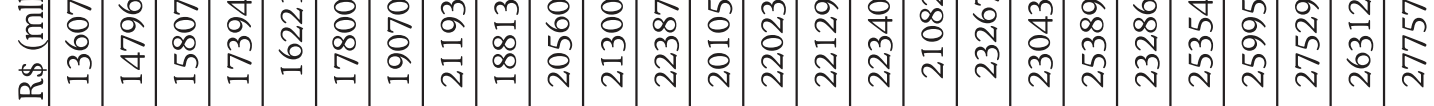

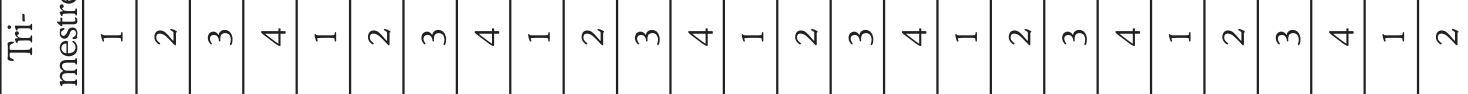

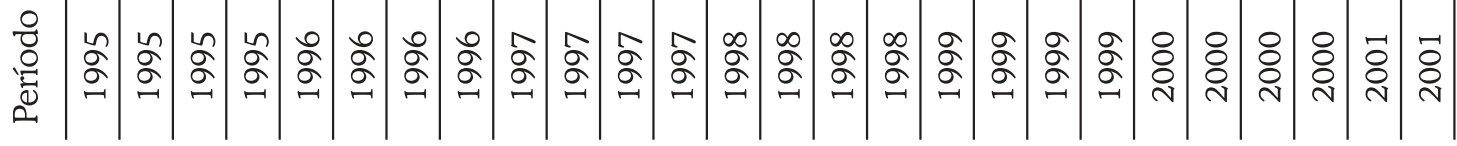




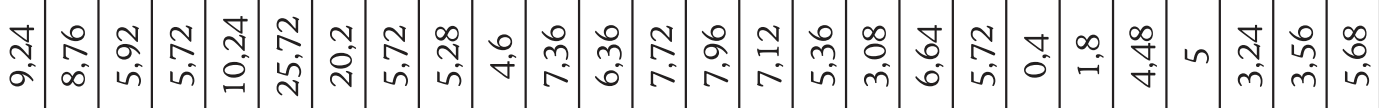

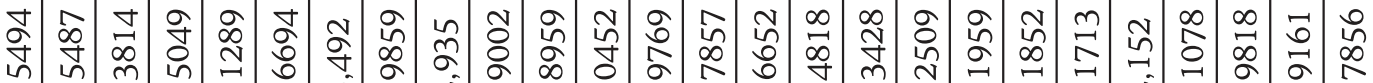

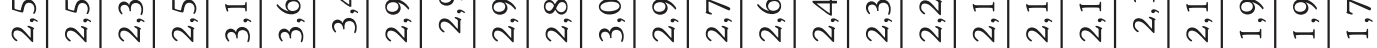

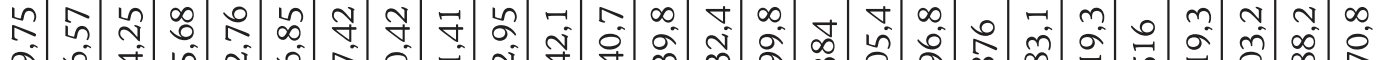
o

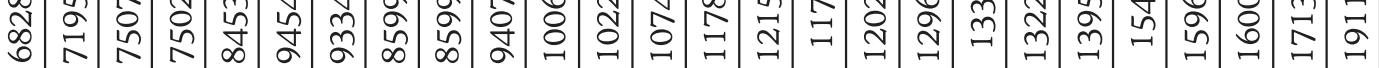

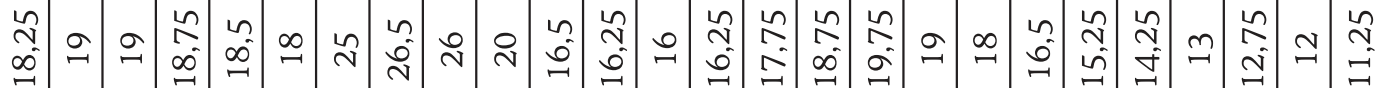

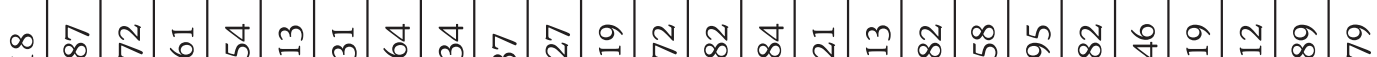

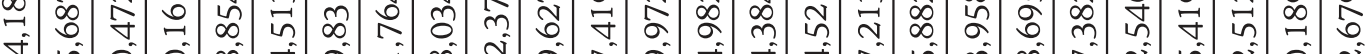

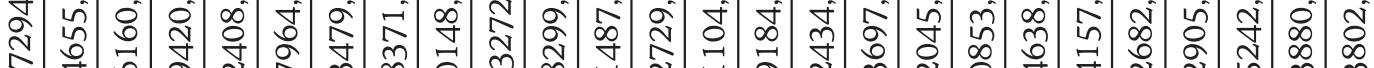

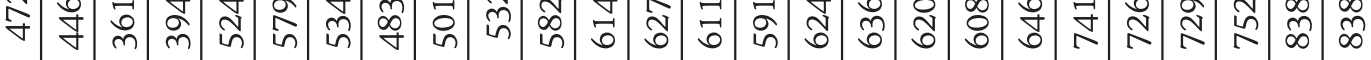

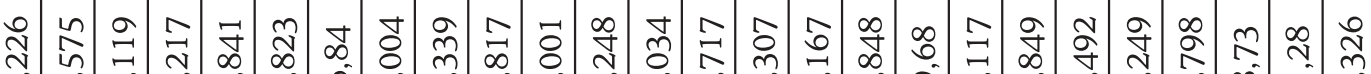

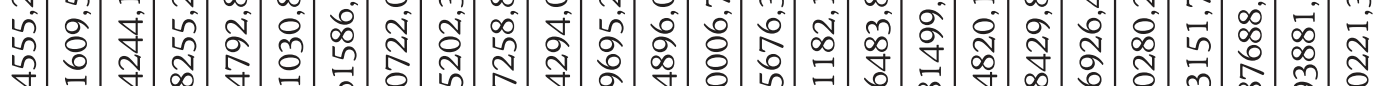

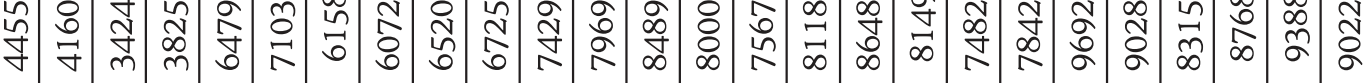

के

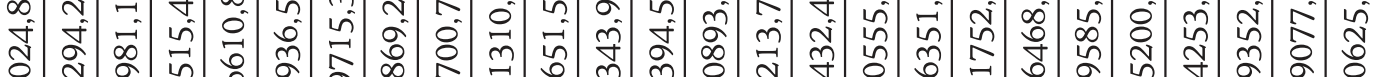

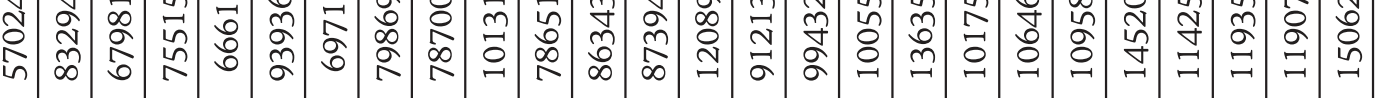

总 g $\stackrel{0}{\rho}$ ริ กิ غ્g $\ddot{0}$ : 융.

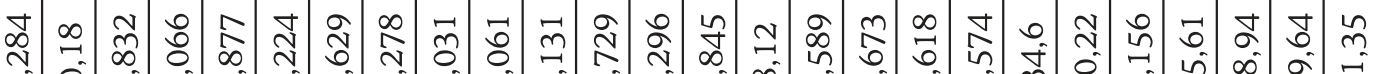

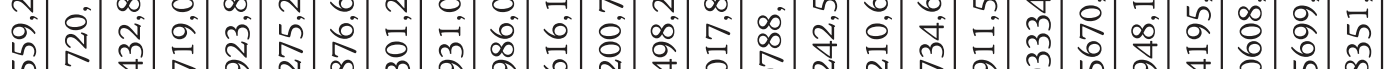

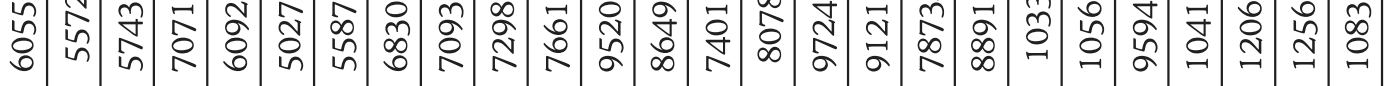

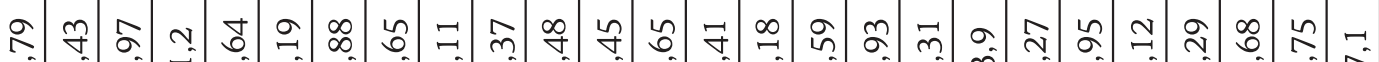

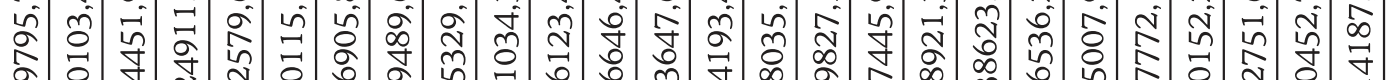

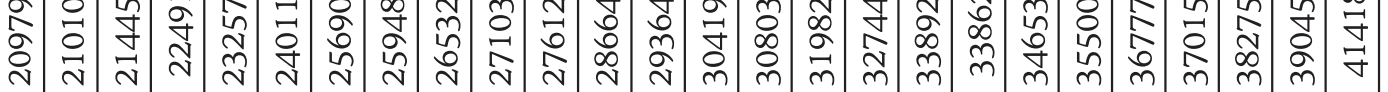

๗ి

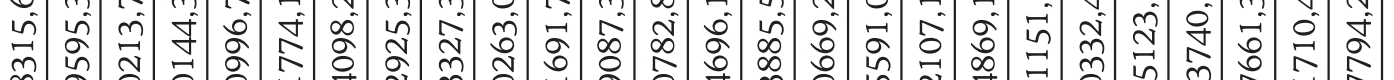

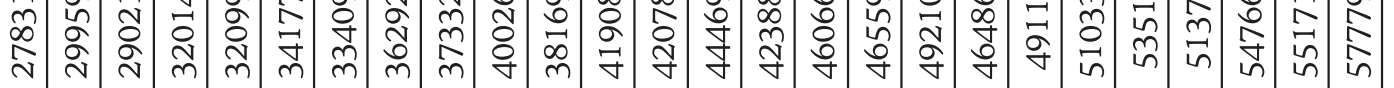

○్

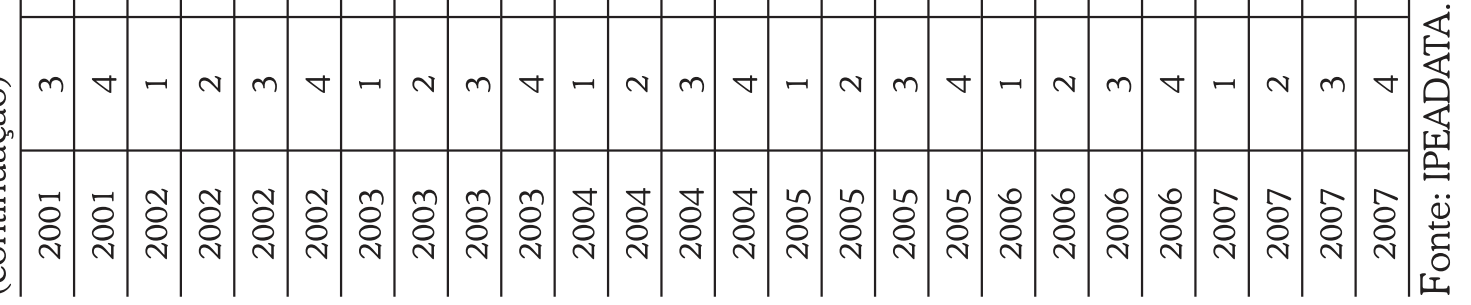

